\title{
Probing UHECR and cosmic ray ensemble scenarios with a global CREDO network
}

\section{Arman Tursunov $^{a, b, *}$ and Piotr Homola ${ }^{c, *}$ on behalf of the CREDO Collaboration} (a complete list of authors can be found at the end of the proceedings)

${ }^{a}$ Research Centre for Theoretical Physics and Astrophysics, Institute of Physics, Silesian University in Opava, Bezručovo nám. 13, CZ-74601 Opava, Czech Republic

${ }^{b}$ Bogoliubov Laboratory for Theoretical Physics, Joint Institute for Nuclear Research, Joliot-Curie street 6, 141980 Dubna, Russia

${ }^{c}$ Institute of Nuclear Physics Polish Academy of Sciences, ul. Radzikowskiego 152, 31-342 Kraków, Poland

E-mail: arman.tursunov@physics.slu.cz

Among theoretical approaches in unveiling the physics of ultra-high-energy cosmic rays (UHECR) one can distinguish the models assuming interactions of exotic super-heavy matter (including extra dimensions, Lorentz invariance violation, cosmic strings, dark matter particles or particles beyond the standard model etc.) and acceleration scenarios describing processes, in which the particles are accelerated by a particular astrophysical object (shocks in relativistic plasma jets, unipolar induction mechanisms, second-order Fermi acceleration, energy transfer from black holes or compact stars etc.). Primary UHECR particles can produce cascades already above the Earth atmosphere, which may be detected as the so-called cosmic ray ensembles (CRE) - the phenomena composed of at least two cosmic ray particles, including photons, with a common primary interaction vertex or the same parent particle with correlated arrival directions and arrival times. In this contribution, we give a brief description of a novel approach to the probing of cosmic ray scenarios with the global Cosmic Ray Extremely Distributed Observatory (CREDO) network.

$37^{\text {th }}$ International Cosmic Ray Conference (ICRC 2021)

July 12 th - 23rd, 2021

Online - Berlin, Germany

\footnotetext{
*Presenter
} 
The standard model of particle physics predicts that if cosmic rays are primarily composed by protons, there should exist a limit on the maximum energy of the particle coming from far distances. This bound is called the GZK cutoff limit [1]. The current experimental data suggests an extragalactic origin for UHECR with energies above the GZK cutoff [2]. Therefore, transient astronomical events are also of the great interest for the emission of tracers of primary UHECR. These include e.g. gamma-ray bursts, supernovae, fast radio bursts, various classes of active galactic nuclei (AGN) with SMBH at their centres, such as Seyfert galaxies, radio galaxies and blazars. Primary UHECR are expected to interact with radiation and matter on their way through the cosmos and give birth to the Cosmic Ray Ensembles (CRE) - the phenomena composed of at least two cosmic ray particles or photons, with a common primary interaction vertex or the same parent particle with correlated arrival directions and arrival times. Such cascades could be formed both within classical models (e.g. products of photon-photon interactions) and exotic scenarios (e.g. result of decay of super-heavy dark matter particles and subsequent interactions). Some of CRE might have a significant spatial extent, which could serve as a unique signature detectable with the existing cosmic ray infrastructure taken as a network of detectors. This signature would be composed of a number of air showers with parallel axes. So far, the cosmic ray research has been oriented on detecting single air showers only, while the search for ensembles of cosmic ray events is a scientific terra incognita.

Experimental searches for cosmic-ray correlations have been realized on different scales with the highest energy events observed by the leading collaborations: Pierre Auger Observatory [3] and Telescope Array [4], as well as the projects with distributed arrays of detectors, such as e.g. CHICOS [5] in the U.S., ALTA [6] in Canada, CZELTA [7] in the Czech Republic, GELATICA [8] in Georgia, EEE [9] in Italy, and LAAS [10] in Japan. Time correlation of registered showers was studied at the distances from $100 \mathrm{~m}$ to $7000 \mathrm{~km}$, and in some cases evidence for unexpected coincidences have been found, however without any convincing follow-up studies and data taking campaigns, which is hard without a global coordination. Only very recently the idea of looking for large scale correlations in a general and global way took shape of the Cosmic Ray Extremely Distributed Observatory (CREDO) Collaboration (see, recent review [11]), formalized in September 2019. CREDO is meant to be a multi-technique (different detector types) and doubly open (for both data upload and offering access) infrastructure enabling a global research programs concerning radiation (both cosmic and terrestrial), with a number of multi-messenger, multi-mission and transdisciplinary opportunities. The CREDO initiative with its objectives dedicated to going beyond studying individual cosmic rays and taking under investigation also UHECR products, may provide a precious complementary approach to UHECR studies. The status of the dispute in the UHECR area encourages a closer look at the field and being ready for a major revision or breakthrough in the understanding of physics at the highest energies known.

To date, most of the data collected by CREDO comes from smartphones with the CREDO Detector app, operating on the Android system with already more than 10.5 million detections, and with the Cosmic Ray App dedicated to iOS devices with more than 7 million detections. Therefore, the work in the direction of science communication, popularization, outreach, education and other activities related to public engagement and citizen science is necessary. Another important example of the infrastructure working within the CREDO Collaboration, although not yet connected to the central system, is HEAMS (High Energy Astrophysics Muon System): an array of muon detectors 
operated by the University of Adelaide, Australia, consisting of several one square meter scintillator muon detectors in two locations distant by $40 \mathrm{~km}$.

In parallel to testing astrophysical scenarios the CREDO Collaboration develops the algorithms that will make us prepared to notice the unexpected physics effects if they come. These algorithms are based on identification of anomalies in the signals received by known and stable detectors. Once cosmic ray anomalies are efficiently identified, they should feed the global multi-messenger and multi-mission programs searching for correlations between different data channels, not only in astrophysics.

Detailed explanation of the CREDO aims, its objectives, methods and tools is given in a recent review [11] and summarized in the proceedings of the ICRC2021 under the article ID 1448 "Invitation to the Cosmic Ray Extremely Distributed Observatory".

\section{Acknowledgments}

A.T. thanks the Institute of Physics of Silesian University in Opava for institutional support. We acknowledge the support from the International Visegrad Fund's Visegrad Grant No. 21920298.

\section{References}

[1] F.W. Stecker. Effect of photomeson production by the universal radiation field on high-energy cosmic rays. Phys. Rev. Lett., 21:1016-1018, 1968.

[2] Pierre Auger Collaboration. Observation of a large-scale anisotropy in the arrival directions of cosmic rays above 8x10 $18 \mathrm{eV}$. Science, 357(6357):1266-1270, Sep 2017.

[3] Pierre Auger Collaboration. The pierre auger cosmic ray observatory. Nucl. Instrum. Methods Phys. Res. A, 798:172-213, 102015.

[4] H. Kawai and et al. Telescope array experiment. Nucl. Phys. B Proc. Suppl., 175:221-226, 2008.

[5] B. E. Carlson, B. Brobeck, and et al. Search for correlated high energy cosmic ray events with CHICOS. Journal of Physics G: Nuclear and Particle Physics, 31(5):409-416, 32005.

[6] S. Pospıšil, P. Pridal, J. Smejkal, R. Soluk, I. Štekl, and V. Vicha. ALTA/CZELTA-a sparse very large air shower array: overview of the experiment and first results. icrc2009.uni.lodz.pl, 2009.

[7] F. Blase, J. Čermák, J. Hubík, P. Lichard, P. Přidal, J. Smejkal, K. Smolek, I. Štekl, and V. Vícha. CZELTA: An overview of the CZECH large-area time coincidence array. Astrophysics and Space Sciences Transactions, 7(1):69-73, February 2011.

[8] M. Svanidze, Y. Verbetsky, and et al. First results obtained by the GELATICA network of the cosmic rays stations in georgia. GESJ: Physics, 1 (5):84-96, 2011.

[9] M Abbrescia and et al. EEE - extreme energy events: an astroparticle physics experiment in italian high schools. J. Phys. Conf. Ser., 718(8):082001, 62016. 
[10] H. Matsumoto and et al. Multiple linsley method for EAS energy determination in LAAS. $J$. Phys. Conf. Ser., 1181(1):012079, 32019.

[11] P. Homola et al. for the CREDO Collaboration. Cosmic-Ray Extremely Distributed Observatory. Symmetry, 12(11):1835, November 2020. 


\section{Full Authors List: CREDO Collaboration}

Arman Tursunov ${ }^{1,2}$ Piotr Homola ${ }^{3}$, David E. Alvarez Castillo ${ }^{3,2}$, Nikolai Budnev ${ }^{4}$, Alok C. Gupta ${ }^{5}$, Bohdan Hnatyk ${ }^{6}$, Marcin Kasztelan $^{7}$, Peter Kovacs ${ }^{8}$, Bartosz Łozowski ${ }^{9}$, Mikhail V. Medvedev ${ }^{10,11}$, Alona Mozgova ${ }^{6}$, Michał Niedźwiecki ${ }^{12}$, Maciej Pawlik ${ }^{13,14}$, Matías Rosas ${ }^{15}$, Krzysztof Rzecki ${ }^{14}$, Katarzyna Smelcerz ${ }^{12}$, Karel Smolek ${ }^{16}$, Jarosław Stasielak ${ }^{3}$, Sławomir Stuglik ${ }^{3}$, Manana Svanidze $^{17}$, Oleksandr Sushchov ${ }^{3}$, Yuri Verbetsky ${ }^{17}$, Tadeusz Wibig ${ }^{18}$, Jilberto Zamora-Saa ${ }^{19}$.

${ }^{1}$ Research Centre for Theoretical Physics and Astrophysics, Institute of Physics, Silesian University in Opava, Bezručovo nám. 13, CZ-74601 Opava, Czech Republic.

${ }^{2}$ Bogoliubov Laboratory for Theoretical Physics, Joint Institute for Nuclear Research, Joliot-Curie street 6, 141980 Dubna, Russia.

${ }^{3}$ Institute of Nuclear Physics Polish Academy of Sciences, Radzikowskiego 152, 31-342 Kraków, Poland.

${ }^{4}$ Irkutsk State University, Russia.

${ }^{5}$ Aryabhatta Research Institue of Observational Sciences (ARIES), Manora Peak, Nainital 263001, India.

${ }^{6}$ Astronomical Observatory of Taras Shevchenko National University of Kyiv, 04053 Kyiv, Ukraine.

${ }^{7}$ National Centre for Nuclear Research, Andrzeja Soltana 7, 05-400 Otwock-Świerk, Poland.

${ }^{8}$ Institute for Particle and Nuclear Physics, Wigner Research Centre for Physics, 1121 Budapest, Konkoly-Thege Miklós út 29-33, Hungary.

${ }^{9}$ Faculty of Natural Sciences, University of Silesia in Katowice, Bankowa 9, 40-007 Katowice, Poland.

${ }^{10}$ Department of Physics and Astronomy, University of Kansas, Lawrence, KS 66045, USA.

${ }^{11}$ Laboratory for Nuclear Science, Massachusetts Institute of Technology, Cambridge, MA 02139, USA.

${ }^{12}$ Department of Computer Science, Faculty of Computer Science and Telecommunications, Cracow University of Technology, Warszawska 24, 31-155 Kraków, Poland.

${ }^{13}$ ACC Cyfronet AGH-UST, 30-950 Kraków, Poland.

${ }^{14}$ AGH University of Science and Technology, Mickiewicz Ave., 30-059 Kraków, Poland.

${ }^{15}$ Liceo 6 Francisco Bauzá, Montevideo, Uruguay.

${ }^{16}$ Institute of Experimental and Applied Physics, Czech Technical University in Prague.

${ }^{17}$ E. Andronikashvili Institute of Physics under Tbilisi State University, Georgia.

${ }^{18}$ University of Łódź, Faculty of Physics and Applied Informatics, 90-236 Łódź, Pomorska 149/153, Poland.

${ }^{19}$ Universidad Andres Bello, Departamento de Ciencias Fisicas, Facultad de Ciencias Exactas, Avenida Republica 498, Santiago, Chile. 